\title{
Next-generation sequencing-based molecular characterization of primary urinary bladder adenocarcinoma
}

\author{
Somak Roy ${ }^{1}$, Dinesh Pradhan ${ }^{1}$, Wayne L Ernst ${ }^{1}$, Stephanie Mercurio ${ }^{1}$, Yana Najjar ${ }^{2}$, \\ Rahul Parikh ${ }^{2}$, Anil V Parwani ${ }^{3}$, Reetesh K Pai ${ }^{1}$, Rajiv Dhir ${ }^{1}$ and Marina N Nikiforova ${ }^{1}$ \\ ${ }^{1}$ Department of Pathology, University of Pittsburgh Medical Center, Pittsburgh, PA, USA; ${ }^{2}$ Department of \\ Medical Oncology and Hematology, University of Pittsburgh Medical Center, Pittsburgh, PA, USA and \\ ${ }^{3}$ Department of Pathology, Ohio State University, Columbus, OH, USA
}

\begin{abstract}
Primary bladder adenocarcinoma is a rare and aggressive tumor with poor clinical outcomes and no standard of care therapy. Molecular biology of this tumor is unknown due to the lack of comprehensive molecular profiling studies. The study aimed to identify genomic alterations of clinical and therapeutic significance using nextgeneration sequencing and compare genomic profile of primary bladder adenocarcinoma with that of high-grade urothelial carcinoma and colorectal adenocarcinoma. A cohort of 15 well-characterized primary bladder adenocarcinoma was subjected to targeted next-generation sequencing for the identification of mutations and copy-number changes in 51 cancer-related genes. Genomic profiles of 25 HGUCs and 25 colorectal adenocarcinomas using next-generation sequencing of 50 genes were compared with primary bladder adenocarcinoma. Genomic profiles were visualized using JavaScript library D3.js. A striking finding was the distinct lack of genomic alterations across the $\mathbf{5 1}$ genes assessed in mucinous subtype of primary bladder adenocarcinoma. Eleven of 15 primary bladder adenocarcinoma harbored at least one genomic alteration in TP53, KRAS, PIK3CA, CTNNB1, APC, TERT, FBXW7, IDH2 and RB1, many of which are novel findings and of potential therapeutic significance. CTNNB1 and APC mutations were restricted to enteric subtype only. While genomic alterations of primary bladder adenocarcinoma showed substantial overlap with colorectal adenocarcinoma, FGFR3 and HRAS mutations and APC, CTNNB1 and IDH2 alterations were mutually exclusive between primary bladder adenocarcinoma and high-grade urothelial carcinoma. These alterations affecting the MAP kinase, PI3K/Akt, Wnt, IDH (metabolic) and Tp53/Rb1 signaling pathways may provide the opportunity for defining targeted therapeutic approaches.

Modern Pathology (2017) 30, 1133-1143; doi:10.1038/modpathol.2017.33; published online 26 May 2017
\end{abstract}

Primary bladder adenocarcinoma is a rare tumor, accounting for $0.5-2 \%$ of all primary bladder malignancies. It usually affects middle age to elderly individuals, with a male preponderance. Enteric, mucinous, signet ring cell and mixed subtypes represent the morphologic heterogeneity in primary bladder adenocarcinoma. ${ }^{1,2}$ These are clinically aggressive tumors that usually present with advanced stage disease at diagnosis with nodal involvement in $30-40 \%$ of the cases and poor

Correspondence: Dr S Roy, MD, Director of Genetics Services and Molecular Informatics, Molecular and Genomic Pathology Laboratory, Department of Pathology, UPMC, 3477 Euler Way, Room\# 7028, Clinical Laboratory Building, Pittsburgh, PA 15213, USA.

E-mail: roys@upmc.edu

Received 25 September 2016; revised 13 March 2017; accepted 13 March 2017; published online 26 May 2017 clinical outcomes unless detected at a very early stage. ${ }^{3,4}$ Unfortunately there exists no effective conventional or targeted therapy for primary bladder adenocarcinoma. Confirming urinary bladder origin of primary bladder adenocarcinoma is a significant diagnostic challenge because of morphologic similarity with primary adenocarcinoma of other sites, such as colorectal and female genital tract. Morphologic and immunophenotypic studies of primary bladder adenocarcinoma have not identified a specific marker. While nuclear expression of betacatenin may suggest a colonic origin, rare cases of primary bladder adenocarcinoma may demonstrate nuclear expression. ${ }^{1,2,5}$ Clinical exclusion of extravesical primary adenocarcinoma is necessary to establish the diagnosis. There is limited data on molecular biology of primary bladder adenocarcinoma and little is known about its genomic profile. 
A few studies have investigated molecular alterations using single gene assay in primary bladder adenocarcinoma to reveal low frequency of KRAS and TERT promoter mutations in $11.5 \%(3 / 26)$ and $28.5 \%$ (4/14) of primary bladder adenocarcinoma, respectively. ${ }^{6,7}$ Although EGFR mutation or amplification and $A L K$ rearrangement was not identified in any primary bladder adenocarcinoma, $36 \%$ of the tumors demonstrated EGFR polysomy. ${ }^{8}$ In this study we aimed to genomically profile a cohort of primary bladder adenocarcinoma using targeted nextgeneration sequencing of 51 cancer-related genes to identify potential therapeutically useful molecular markers. In addition, we compare the targeted molecular profile of primary bladder adenocarcinomas with colorectal adenocarcinoma and high-grade urothelial carcinoma.

\section{Materials and methods}

\section{Sample Cohort}

After approval form the Institutional Review Board at The University of Pittsburgh, the institutional archive of the Department of Pathology was retrospectively reviewed from 1999 to 2016 . We used the World Health Organization (WHO) 2016/International Society of Urologic Pathology definition of primary bladder adenocarcinoma as inclusion criteria. The cases of urothelial carcinoma with focal or extensive glandular differentiation as well as those with clinical evidence of metastasis from other sites, such as colorectal or female genital tract and urachal adenocarcinomas were excluded. This yielded a total of 15 cases of primary bladder adenocarcinoma, which had adequate and viable tumor content for molecular analysis. For comparative analysis, genomic data from consecutive 25 cases of high-grade urothelial carcinoma without adenocarcinoma component and consecutive 25 cases of colorectal adenocarcinoma that were received for routine clinical molecular testing, were retrospectively retrieved from our laboratory information system (LIS). Data on TERT promoter sequencing was not available for these 50 clinical cases. All histologic slides including Hematoxylin and Eosin (H\&E) and Immunohistochemistry were reviewed and a specific formalin-fixed paraffin embedded tissue block was selected for molecular analysis. Paired normal tissue was not used sequencing analysis.

\section{Targeted Next-Generation Sequencing}

Next-generation sequencing analysis was performed in a Clinical Laboratory Improvements and Amendments (CLIA)-certified molecular laboratory. Tumorrich areas ( $>50 \%$ neoplastic cells), targeted using Hematoxylin and Eosin (H\&E) stain guidance by a pathologist, were microdissected using eight to ten, $4-\mu \mathrm{m}$ unstained histological sections. Genomic DNA was isolated from each target with the DNeasy Blood \& Tissue Kit on the automated QIAcube (Qiagen, CA, USA) instrument according to the manufacturer's instructions and amplified using the Ion AmpliSeq Library Kit 2.0 and Ion AmpliSeq Cancer Hotspot Panel v2 primer pool (Life Technologies, Carlsbad, CA, USA) covering hotspots across 50 cancer-related genes (Supplementary Table 1). Subsequently, the multiplexed, barcoded libraries were enriched by clonal amplification and massively parallel sequencing was carried out on an Ion Proton sequencer (Life Technologies). After a successful sequencing reaction, the raw signal data were analyzed using Torrent Suite v4.2.1 (Life Technologies). The short sequence reads were aligned to the human genome reference sequence (GRCh37 patch 13, GCF_000001405.25). Variant calling was performed using Variant Caller v4.4.3.3 plugin (integrated with Torrent Suite) that generated a list of identified sequence variations (single-nucleotide variants (SNV) and insertions or deletions (indels)) in a variant calling file (VCF v4.2; https://samtools.github. io/hts-specs/VCFv4.2.pdf). Copy-number analysis from next-generation sequencing data was performed as previously described. ${ }^{9}$ The variant calls were annotated using inhouse developed custom software. Subsequently, HGVS variant nomenclature was normalized and variants prioritized using SeqReporter. ${ }^{10}$ An in-house knowledge base and several publically available databases were used (Supplementary Methods) for variant annotation. Sequence variants with at least $5 \%$ allelic fraction with at least $300 \times$ depth of coverage or $3 \%$ allelic fraction with at least $1000 \times$ depth of coverage were included for analysis. Integrated Genomics Viewer (IGV; Broad Institute, Cambridge, MA, USA) was used for manual review of the sequence read pileups. Visualization plots were created using javascript library D3.js (https://d3js.org/; last accessed 29 May 2016). The TERT promoter was amplified by PCR on extracted DNA using and interrogated for the hotspot mutation loci for g.1295228C $>$ T (c.-124C $>$ T; C228T) and g.1295250C $>$ T (c.-146C $>$ T; C250T) using bi-directional Sanger sequencing (supplementary Material).

\section{Immunohistochemistry}

Immunohistochemistry for $\beta$-catenin, MLH1, PMS2, MSH2 and MSH4 were performed on all cases of primary bladder adenocarcinoma (Supplementary Table 2). Briefly, formalin-fixed paraffin embedded sections were cut at $4 \mu$, deparaffinized and hydrated. Subsequent to heat induced epitope retrieval and endogenous peroxidase quenching, slides were incubated with primary antibody for 16-30 min depending on the antibody, followed by secondary antibody (Mach 2 Mouse HRP, Biocare, Concord, CA, USA) for $30 \mathrm{~min}$ and substrate chromogen (DAKO, Carpinteria, CA, USA) for $5 \mathrm{~min}$. Slides were washed 
with TBS buffer and finally counterstained with Harris hematoxylin (Supplementary Methods).

\section{Results}

\section{Clinicopathologic Findings}

Table 1 summarizes the clinicopathologic findings of this cohort of primary bladder adenocarcinoma (Supplementary Table 3 for details). Posterior wall was the most frequently involved site $(12 / 15,80 \%)$. Smoking status was available for 11 of the 15 patients, of which 6 patients $(\sim 55 \%)$ were smokers and did not correlate with genomic profile or other clinicopathologic parameters. The majority of the primary bladder adenocarcinomas demonstrated aggressive clinicopathologic features; Five of the 10 $(50 \%)$ enteric-type primary bladder adenocarcinomas and all mucinous and signet ring cell type primary bladder adenocarcinomas were high stage (pT3 or pT4). 6 patients had nodal disease (pN2), all of which were either stage pT3 (1/6) or pT4 (5/6), and 5 patients harbored visceral metastasis involving the lung, liver, bone, pleura, pericardium or peritoneum. Clinical follow-up was available for 14 of the 15 patients (median follow-up of 33.5 months, range 2-132 months). Six patients died of extensive and recurrent disease and 3 patients were alive with tumor at the time of last follow-up.

\section{Genomic Alterations}

Histologic tumor sections were microdissected with at least $60 \%$ tumor cellularity. Quality and quantity of extracted DNA and next-generation sequencing DNA libraries were adequate. The depth of coverage across the sequenced targeted regions was uniform with average hotspot coverage of $3739 \times$ per sample (Supplementary Figure 1). Figure 1 summarizes the overall genomic alterations in context of clinicopathologic features of 15 primary bladder adenocarcinoma in this cohort. Eleven of 15 primary bladder adenocarcinomas (73\%) harbored at least one genomic alteration. The TP53 gene was most frequently altered $(47 \%)$ followed by PIK3CA $(20 \%)$, and KRAS $(20 \%)$. Several clinically relevant and actionable genomic alterations were identified, many of which are characteristic of other tumor types but novel in primary bladder adenocarcinoma (Supplementary Table 4). The following activating oncogenic alterations were identified; KRAS (p.G12A, p.G12D and p. G13D), PIK3CA (p.E542K, p.C420R, p.N1044K), CTNNB1 (p.D32N, p.S45F and gene amplification), IDH2 (p.R172S), and TERT promoter (c.-124C > T/ C228T). TERT promoter mutation was identified in 2 of $15(13 \%)$ cases only, one of which demonstrated an enteric morphology, while the other case had a predominance of signet ring cells (Figure 2). The latter case of signet ring cell also harbored a PIK3CA mutation (p.C420R).
Table 1 Clinicopathologic profile of 15 primary bladder adenocarcinoma cohort included in this study

\begin{tabular}{lc}
\hline Clinicopathologic features & $\mathrm{N}(\%)$ \\
\hline Age, mean (range) (years) & $66(53-97)$ \\
Sex & \\
Male & $10(67)$ \\
Female & $5(33)$ \\
Bladder wall location & \\
Posterior & $12(80)$ \\
Anterior & $3(20)$ \\
& \\
Tumor size (mean) (range) (cm) & \\
& $4(0.8-7.5)$ \\
Histologic subtype & \\
Enteric & $9(60)$ \\
Mucinous & $3(20)$ \\
Signet ring cell & $2(14)$ \\
Enteric+Mucinous & $1(7)$ \\
pT stage & \\
pT1 & $1(7)$ \\
pT2 & $5(33)$ \\
pT3 & $4(27)$ \\
pN (nodal status) & $7(66)$ \\
N0 & \\
N1 & \\
N2 & \\
pM (metastasis) & $9(60)$ \\
M0 & $0(0)$ \\
& \\
\hline & \\
&
\end{tabular}

${ }^{a}$ In 1 case tumor size was not available because resection was not performed and patient was lost to follow-up. In another case, the tumor was multifocal ranging in size from 0.3 to $0.7 \mathrm{~cm}$.

Upon correlation with clinicopathologic features of these tumors, primary bladder adenocarcinoma with predominantly mucinous morphology did not harbor any genomic alterations in the targeted regions of the 51 genes. In addition, alterations in genes of the Wnt pathway (APC or CTNNB1) were restricted to tumors with enteric morphology only. Mutation profile did not correlate with other clinicopathologic parameters.

\section{CTNNB1, APC Mutations and $\beta$-Catenin Immunoexpression}

Primary bladder adenocarcinoma harbored CTNNB1 $(3 / 15 ; 20 \%)$ and $A P C(2 / 15 ; 13 \%)$ mutations that were restricted to tumors with enteric morphology. APC mutations were truncating (p.E1309Dfs* 4 and p.R876*), that were likely to result in a truncated and non-functional APC protein. In contrast, CTNNB1 alterations included activating missense mutations at known hotspots (p.D32N and p.S45F) and amplification. $A P C$ and $C T N N B 1$ alterations were mutually exclusive with the exception of one primary bladder adenocarcinoma (case \#5), where a truncating mutation in $A P C$ and activating missense mutations 


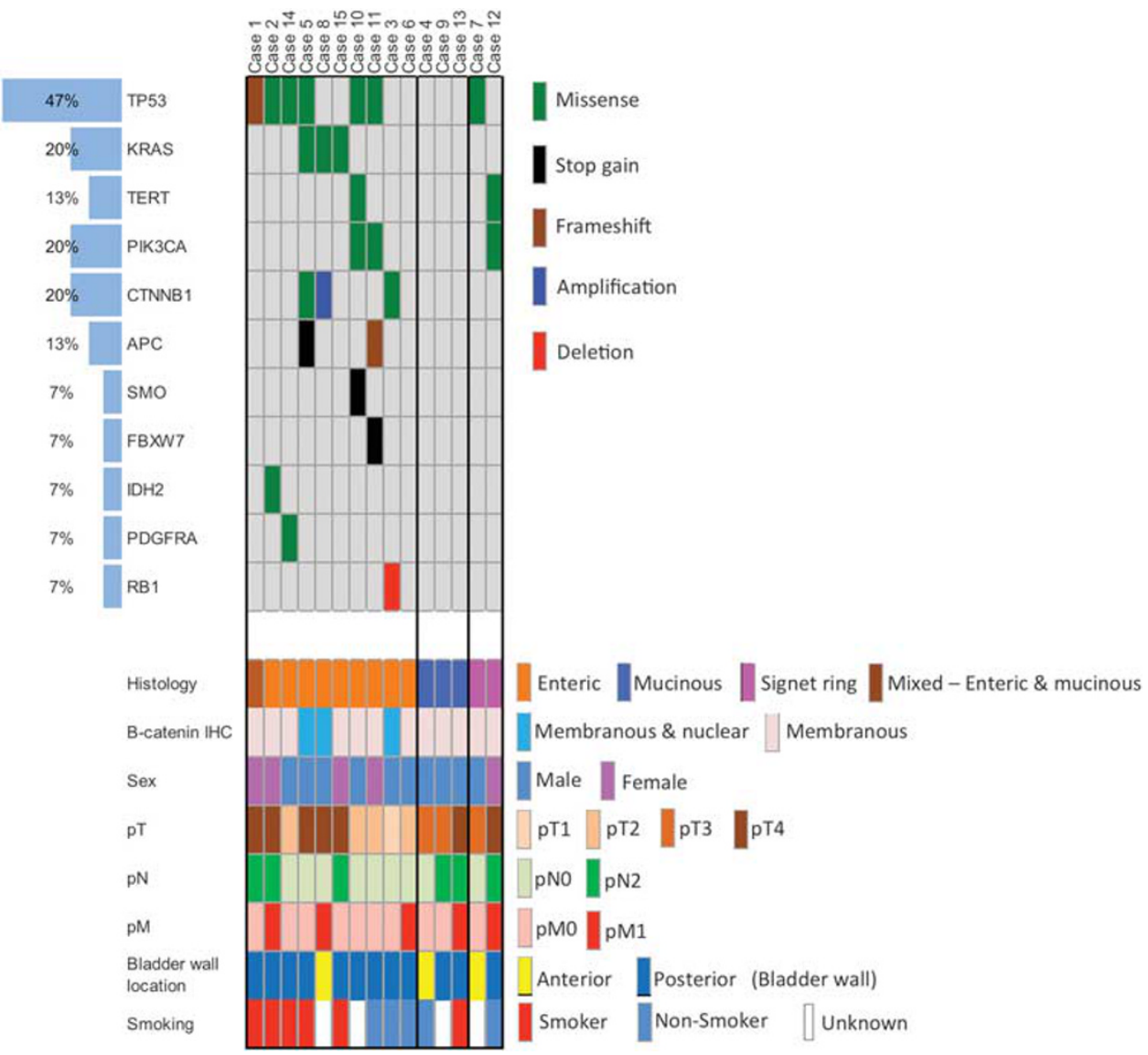

Figure 1 Comutation plot summarizing the genomic alterations for the 15 cases of primary bladder adenocarcinoma and mapping them to various clinicopathologic parameters (bottom tracks).

in CTNNB1 genes were noted. Upon comparison with $\beta$-catenin immunoexpression, nuclear expression was restricted to cases harboring $A P C$ or CTNNB1 alterations $(n=3)$. In one case (case 11), which harbored a truncating $A P C$ mutation (p.E1309 $\mathrm{Dfs} * 4$ ), nuclear expression of $\beta$-catenin was not identified. In contrast, TERT promoter and IDH2 mutations were identified in cases without Wnt pathway gene alterations and membranous $\beta$-catenin expression. In addition, immunohistochemical stains for DNA mismatch repair proteins MLH1, PMS2, MSH2 and MSH6 were performed on all cases of primary bladder adenocarcinoma, and revealed no evidence of deficient expression.

\section{Genomic Alterations in Colorectal Adenocarcinoma}

Twenty-four of the 25 cases of advanced stage colorectal carcinomas harbored at least one genomic alteration. The most frequently altered genes included TP53 $(17 / 25 ; 68 \%), A P C(13 / 25 ; 52 \%)$, KRAS $(13 / 25 ; 52 \%)$ and SMAD4 (8/25; 32\%). Clinically important oncogenes such as PIK3CA, GNAS, BRAF and EGFR were less frequently altered (Supplementary Figure 2a; Supplementary Table 5).

\section{Genomic Alterations in High-Grade Urothelial Carcinoma}

Twenty-three of 25 advanced stage high-grade urothelial carcinoma harbored at least one genomic alteration. TP53 (14/25; 56\%), FGFR3 (7/25; 28\%), PIK3CA $(6 / 25 ; 24 \%)$ and $R B 1(4 / 25 ; 16 \%)$ were most frequently altered. Other clinically relevant genomic alterations, albeit at a lower prevalence, were detected in FBXW7, HRAS, ERBB2, ATK1 and KRAS (Supplementary Figure 2b; Supplementary Table 6).

\section{Discussion}

Primary adenocarcinoma of the urinary bladder is a rare but aggressive tumor that usually presents at an advanced stage, often with metastatic disease. There is lack of rigorous data on definitive therapy and therefore no standard of care for management of patients with primary bladder adenocarcinoma. These tumors are often treated by extrapolating data from urothelial carcinoma and adenocarcinoma of other primary sites, which are largely ineffective. Patients with non-urachal primary bladder adenocarcinoma are typically treated with radical cystectomy and bilateral pelvic lymph node dissection, ${ }^{11}$ as 

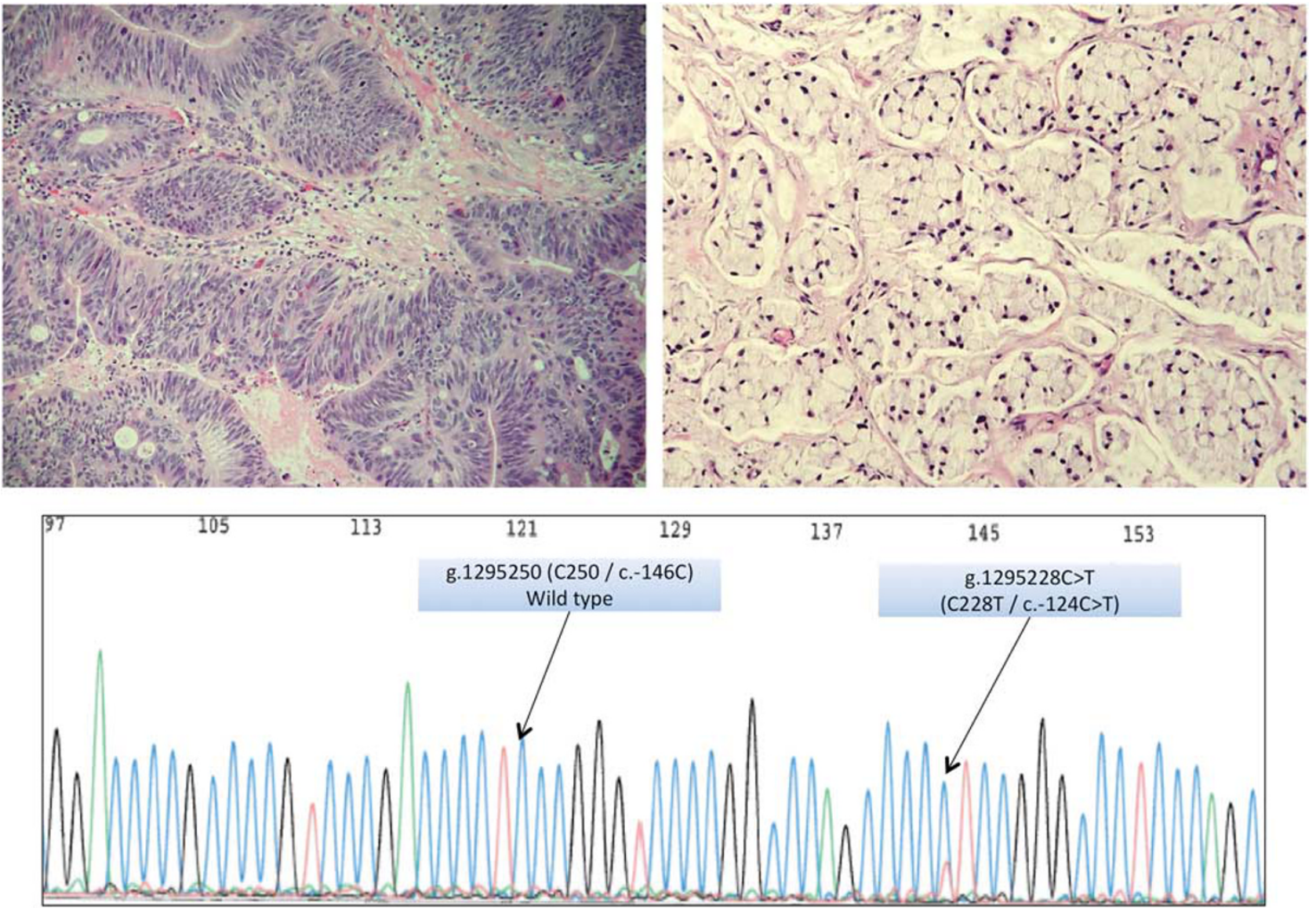

Figure 2 This composite image shows two cases that harbored TERT promoter mutation, as shown in the bottom Sanger electropherogram. Top left and top right images represent predominance of enteric and signet ring cell morphologies for each of the two primary bladder adenocarcinoma cases, respectively (Hematoxylin \& Eosin, $100 \times$ ).

partial cystectomy results in poor outcomes. Neoadjuvant and adjuvant chemotherapy or radiation are not generally recommended, as primary bladder adenocarcinoma was not included in the phase III trial that lead to standard of care neoadjuvant chemotherapy for urothelial bladder carcinoma. The role of chemotherapy and/or radiation is not clear for patients with unresectable or metastatic disease, and there have been no completed randomized trials. Response rates as high as 33\% were seen in patients with urachal adenocarcinomas after treatment with 5-FU and cisplatin combinations in a retrospective study on 42 patients, done at $\mathrm{MD}$ Anderson Cancer Center. ${ }^{12}$ In a prospective study of ifosfamide, paclitaxel and cisplatin in 20 men with non-urothelial bladder cancer (11 of whom had primary bladder adenocarcinoma, six with urachal adenocarcinoma), response rate was $36 \%$ and median survival 25 months. ${ }^{13}$ The same response rate was reported in a retrospective analysis of 21 patients who received cisplatin containing first line regimens, including 14 with primary bladder adenocarcinoma. ${ }^{14}$ Other agents have been reported on a case report basis, including 5-FU alone, 5-FU with doxorubicin and mitomycin-c, cisplatin with
S-1 (a novel 5-FU derivative) and combination of folinic acid with 5-FU, oxaliplatin (FOLFOX) and bevacizumab, with variable responses (either poor or prolonged). ${ }^{15-18}$ To our knowledge, there is no current data on the use of targeted agents in bladder adenocarcinoma. In addition, primary bladder adenocarcinoma is a challenging pathologic diagnosis given the significant morphologic and immunophenotypic overlap with the more common metastatic colorectal adenocarcinoma. While most prior studies have focused on addressing this diagnostic challenge using an array of several immunohistochemical markers, there is very limited understanding of the genomic landscape of primary bladder adenocarcinoma, with the exception of single gene/hotspot mutation analysis for KRAS, TERT, and EGFR and $E M L 4 / A L K$ fusions detection by fluorescence in situ hybridization (FISH). ${ }^{6-8,19}$ Given the poor clinical outcomes, these tumors need to be investigated for therapeutically targetable molecular alterations needed to improve patient outcomes.

To the best of our knowledge, this is the first study that has characterized primary bladder adenocarcinoma at a molecular level using a targeted nextgeneration sequencing approach for key cancer 
genes. When stratified based on morphologic subtype, the key observation was the complete absence of mutations or copy-number changes in mucinoustype primary bladder adenocarcinomas in the targeted genes. Further stratification based on $\beta$-catenin nuclear staining was noted to be restricted to the enteric subtype. In our cohort of 15 cases, we identified several clinically significant genomic alterations that are novel in primary bladder adenocarcinoma. Importantly, based on the genomic profile, MAP kinase, mTOR, Wnt and Tp53/Rb1 pathways appear to be involved in the pathogenesis of primary bladder adenocarcinoma. TP53 mutation was the most frequent alteration, majority of which were inactivating missense mutations $(6 / 7 ; 86 \%)$. Three PIK3CA mutations p.E542K, p.C420R and p.N1044K were identified in this cohort. E542K is one of the three well-described hotspot mutations in PIK3CA. Dogruluk et al. demonstrated that many rare PIK3CA non-hotspot mutations are oncogenic (activating), using several in-vitro functional studies and animal model studies. Both the C420R (C2 domain) and N1044K (kinase domain) mutations were shown to be activating, albeit at an intermediate to lower level when compared to the hotspot mutations. ${ }^{20,21}$ PIK3CA mutations have been shown to activate the PI3-kinase pathway and therefore tumors harboring such mutations may respond to mTOR inhibitors such as Everolimus and Temsirolimus.. ${ }^{20}$ In celllines, a novel AKT inhibitor AZD5363 demonstrated significant activity in the presence of PIK $3 C A$ mutations $^{22}$ and is currently in phase II studies for patients harboring PIK3CA mutations or amplification (NCT02451956).

IDH2 mutation, p.R172S, was identified in one primary bladder adenocarcinoma (case 2) that also harbored a concomitant TP53 mutation. The IDH2 gene is frequently mutated in acute leukemia and gliomas at the arginine residues, R140 and R172 (substrate-binding site). ${ }^{23,24}$ IDH2 mutations have not been reported in urothelial carcinomas or specifically in primary bladder adenocarcinoma. Mutations in IDH1 and IDH2 in the substratebinding site lead to the abnormal accumulation of 2-hydroxyglutarate. This in turn results in upregulation of proto-oncogenes such as HIF-1alpha, histone modification and chromatin remodeling and alterations of the cellular microenvironment. ${ }^{24}$ IDH hotspot mutations are of potential therapeutic importance. Early phase $1 / 2$ and phase 3 clinical trials are currently underway investigating the role of targeted therapeutic agents (AG-881, AG-221) in patients with advanced solid tumors and hematologic malignancies that harbor IDH2 mutations (NCT02577406, NCT02481154, NCT02492737).

One of the primary bladder adenocarcinomas (case 11) harbored a FBXW7 stop gain mutation (p.R278*). Unlike the common hotspots (R465, R479 and R505) in the WD repeat domain, this mutation maps to the upstream F-box-like domain and results in a truncated, non-functional protein. ${ }^{25,26}$ FBXW7 gene is located on chromosome 4 q31.3 and is a major tumor suppressor gene that negatively regulates several key downstream cellular pathways such as cell cycle control, DNA damage and cellular apoptosis, and epithelial-mesenchymal transition. Important oncogenic proteins such as c-Myc, c-Jun, cyclin E1, KFL5, mTOR and Notch 1 have been shown to be substrates for fbxw7 protein. Loss of function of $F B X W 7$ via truncating mutations or copy-number loss has been documented in up to $30 \%$ human cancers with T-ALL and cholangiocarcinoma harboring the highest frequency of $F B X W 7$ alterations. ${ }^{25}$ It is rare in urothelial carcinoma and has not been reported previously in primary bladder adenocarcinoma. Similar to prior studies in non-urothelial tumors, the primary bladder adenocarcinoma case with FBXW7 mutation in our cohort also harbored concomitant mutations in TP53, PIK3CA and APC genes.

One of the most intriguing and novel finding in our cohort was the presence of $A P C$ and/or CTNNB1 mutations in primary bladder adenocarcinoma. The results of several previous studies have substantiated that the membranous pattern of $\beta$-catenin expression, first described by Wang et al., is the only reliable marker for supporting the diagnosis of primary bladder adenocarcinoma against metastatic colorectal adenocarcinoma in most, but not all cases. ${ }^{1,2,5}$ In a small subset of colorectal adenocarcinoma, nuclear $\beta$-catenin staining is not seen. Conversely, a small proportion of primary bladder adenocarcinomas demonstrates abnormal nuclear expression of $\beta$-catenin. ${ }^{1,2}$ Therefore, clinical correlation is necessary to establish or refute the diagnosis of primary bladder adenocarcinoma. This unusual $\beta$-catenin expression profile has not been correlated with molecular findings in any prior studies. Our cohort harbored truncating mutations in $A P C$ and activating CTNNB1 ( $\beta$-catenin) mutations in 4 of 15 cases $(27 \%)$. Three of these 4 cases that demonstrated nuclear $\beta$-catenin staining in a subset of tumor cells (10-50\%) were found to harbor CTNNB1 mutation only (p.D32N), CTNNB1 mutation (p.S45F) with $A P C$ mutation (p.R876*) and CTNNB1 amplification (Figure 3).

The Wnt signaling pathway has a critical role in tumorigenesis of many different cancer types. $\beta$-catenin is a transcriptional co-activator, which when translocated inside the nucleus activates the Wnt pathway by forming complexes with LEF/TCF family of transcription factors. APC-axin-axin2 complex mediated proteosomal degradation in the cytoplasm by ubiquitination is the principal regulatory mechanism for $\beta$-catenin. Glucose synthase kinase $3 \beta$ (GSK3 $\beta$ ) mediated phosphorylation of the N-terminal serine/threonine amino acid residues primes $\beta$-catenin for ubiquitination. ${ }^{27}$ Excessive cytoplasmic accumulation of $\beta$-catenin due to failure of the regulatory mechanism results in nuclear translocation and Wnt pathway activation. While inactivation of APC is the most common mechanism of Wnt pathway upregulation, activating mutations 


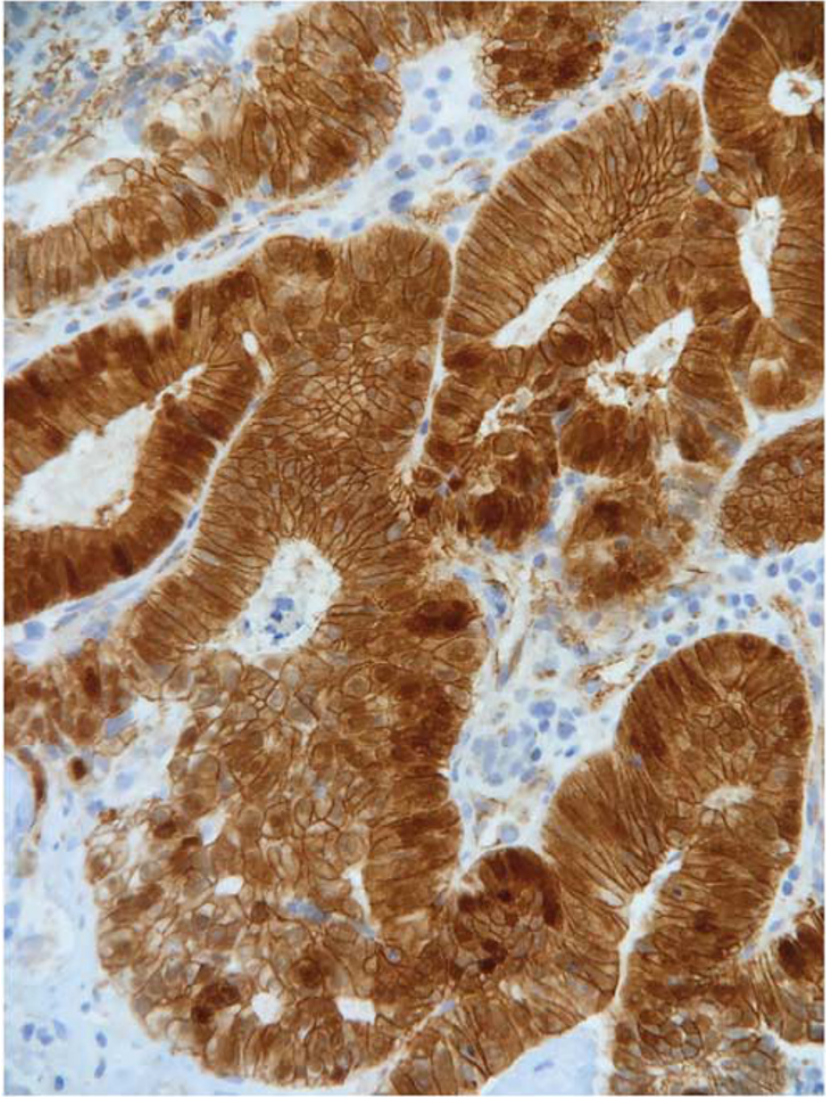

APC or CTNNB1 alteration positive

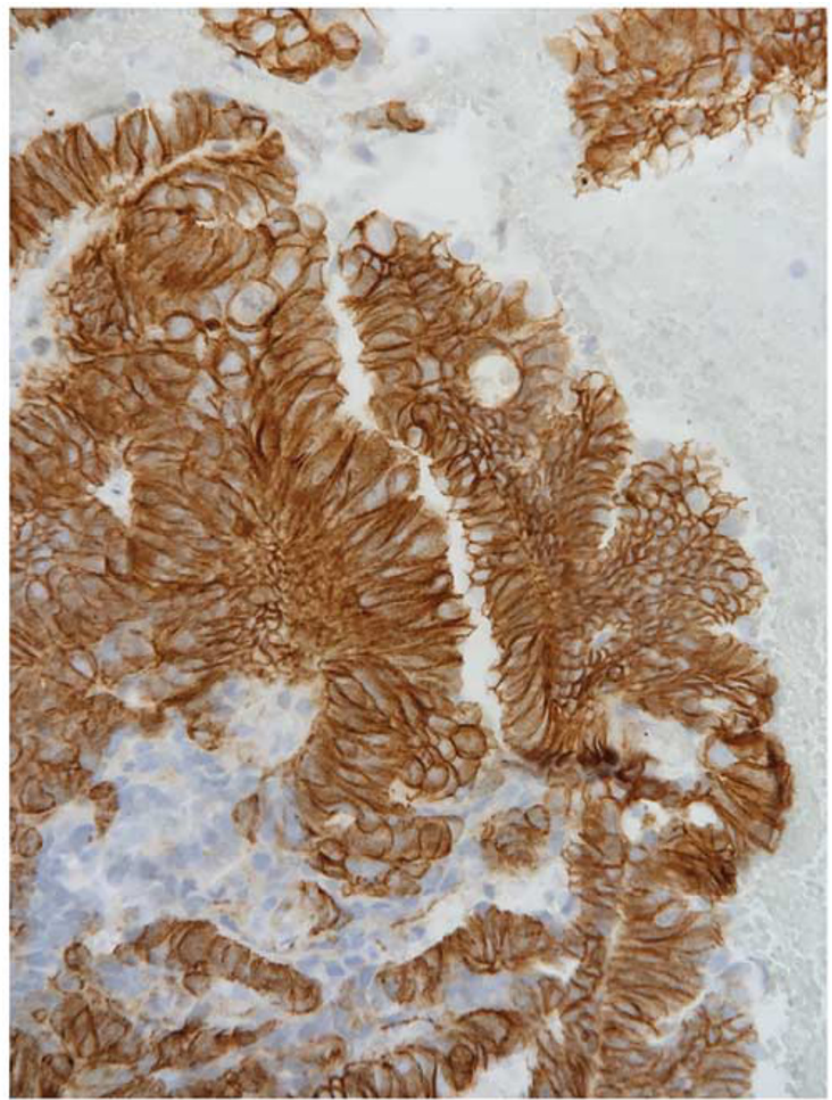

APC or CTNNB1 alteration negative

Figure 3 Photomicrographs demonstrating the presence (left) and absence (right) of nuclear $\beta$-catenin localization in primary bladder adenocarcinomas with and without APC or CTNNB1 alterations (DAB Chromogen, $200 \times$ ).

in exon 3 of CTNNB1 gene is a well-documented alternative mechanism. The $\mathrm{S} 45 \mathrm{~F}$ and $\mathrm{D} 32 \mathrm{~N}$ are common hotspot mutations in CTNNB1 that affect the GSK3 $\beta$ phosphorylation and APC binding domain resulting in stabilization of $\beta$-catenin and abnormal cytoplasmic accumulation. ${ }^{27,28}$ CTNNB1 amplification, as seen in one of our cases, is a rare but previously described mechanism that potentially results in $\beta$-catenin overexpression. ${ }^{29}$ In our case, we observed overexpression and abnormal nuclear localization of $\beta$-catenin by immunohistochemistry, confirming the downstream effect of CTNNB1 amplification.

Finally, case 11 harbored a frameshift deletion (p. E1309Dfs*4) in $A P C$ gene but interestingly did not demonstrate abnormal nuclear localization of $\beta$-catenin (Figure 4). The sample was found to have preserved expression of MLH1, PMS2, MSH2 and MSH6 and did not harbor any alterations in the $\beta$-catenin gene. This interesting and unusual finding may be explained as follows: the binding of APC to $\beta$-catenin is a complex key event for nuclear export and subsequent degradation of $\beta$-catenin. Subsequent to phosphorylation of the serine/threonine residue in the $\mathrm{N}$-terminal of $\beta$-catenin, the nuclear export sequence domain in the $\mathrm{N}$-terminal region of the APC protein facilitates nuclear export of $\beta$-catenin into the cytoplasm. ${ }^{30}$ A 20 amino acid repeat region immediately downstream to the nuclear export sequence domain has been postulated to interact with the Axin-APC-GSK3-CK1 complex, facilitating the degradation of cytoplasmic $\beta$-catenin. ${ }^{30,31}$ The E1309Dfs* 4 mutation is common mutation in colorectal cancer that results in a truncated APC protein where the nuclear export sequence domain is intact, retaining the capacity for nuclear $\beta$-catenin export, but the 20 amino acid repeat sequence is lost, therefore limiting the ability to subsequently degrade $\beta$-catenin. ${ }^{32}$ This explains our current finding of the lack of nuclear expression of $\beta$-catenin by immunohistochemistry despite an $A P C$ mutation. Other possible explanations include the presence of an intact second $A P C$ allele and alternative mechanisms of $\beta$-catenin nuclear export and degradation that do not require $A P C$. While a loss of $A P C$ was not identified using copy-number analysis, biallelic inactivation by copy neutral loss of heterozygosity (uniparental disomy) or the role of alternative $\beta$-catenin regulators cannot be excluded from our data. Given the complexity of $\beta$-catenin regulation, the ultimate impact of this $A P C$ mutation in Wnt pathway activation remains unknown and will require further studies to investigate it. 

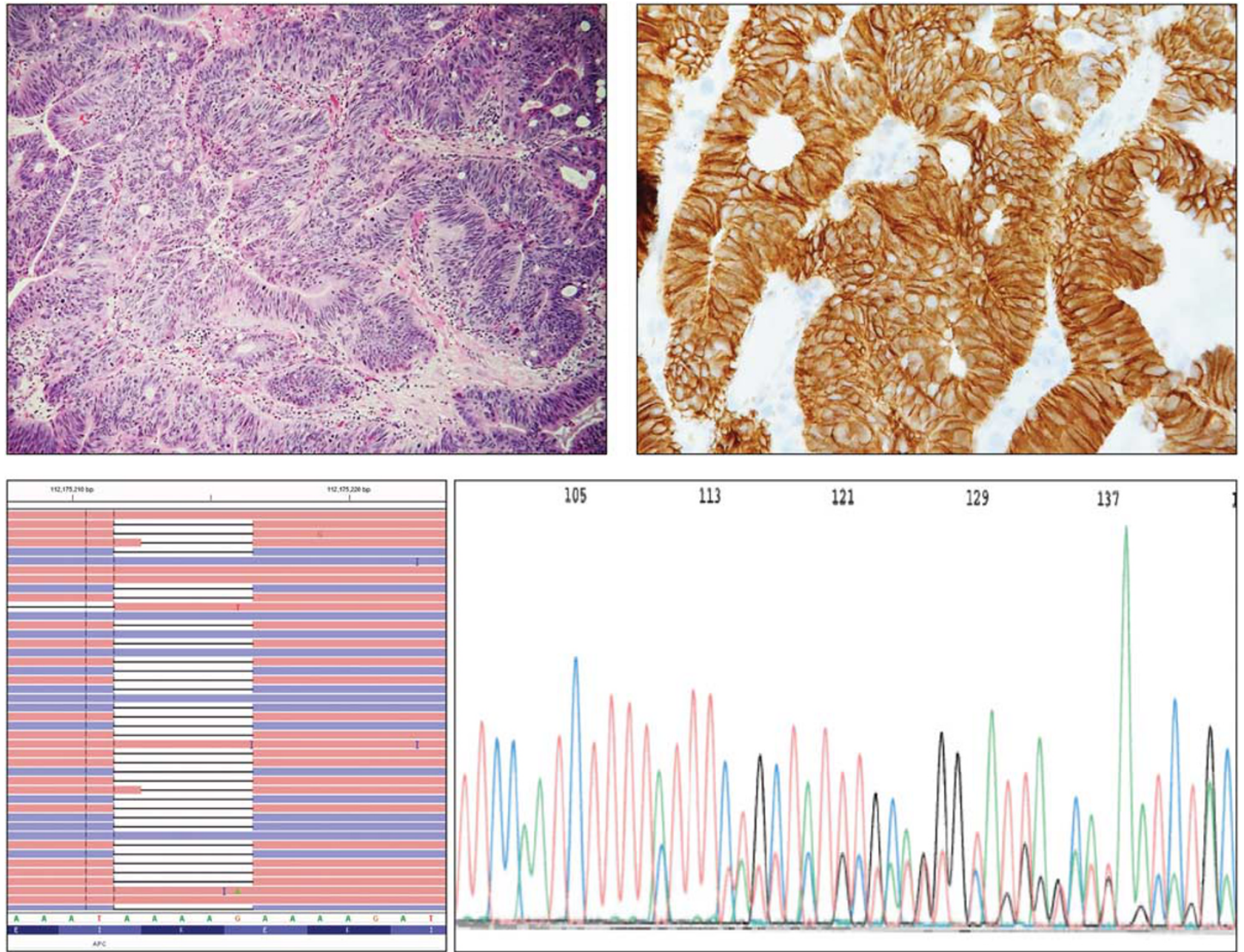

Figure 4 Enteric-type primary bladder adenocarcinoma (case \#11) harboring a APC frameshift mutation, p. E1309Dfs*4, (sequence pileup in IGV—bottom left) confirmed by Sanger sequencing (bottom right) that did not show abnormal nuclear localization of $\beta$-catenin by immunohistochemistry (top right). (Hematoxylin \& Eosin, $100 \times$ and DAB chromogen, $200 \times$ ).

Several synthetic small molecules and peptides are under active investigation as Wnt pathway inhibitors, with promising results in animal models. However, clinical evidence for such targeted therapy is yet to be demonstrated. Given the complex interactions involved in $\beta$-catenin regulation, single agent targeted therapy is unlikely to be effective. ${ }^{33}$

TERT promoter mutation is one of the most common genomic alterations in non-invasive, superficial and muscle invasive urothelial carcinoma. ${ }^{34}$ While prevalence is particularly high in certain subtypes of urothelial carcinoma such as micropapillary, ${ }^{35}$ small cell ${ }^{36}$ and nested, ${ }^{37}$ there are conflicting reports on TERT mutation status in primary bladder adenocarcinoma., ${ }^{79}$ Cowan et al. demonstrated TERT promoter mutation in $28.5 \%$ (4/14) cases using the SafeSeq sequencing technology. All TERT promoter mutations in their study were C228T $(-128 \mathrm{C}>\mathrm{T})$, and interestingly were restricted to tumors with non-enteric morphology only. While the authors included urachal adenocarcinomas in their cohort, only non-urachal primary bladder adenocarcinomas harbored TERT promoter mutation. ${ }^{7}$ In contrast, Vail et al investigated TERT promoter mutation in a variety of neoplastic and non-neoplastic glandular lesions in the urinary bladder, of which none of the 10 primary bladder adenocarcinomas harbored TERT promoter mutation, using the Sanger sequencing approach. ${ }^{19}$ In our cohort, TERT promoter mutation (C228T) was identified in 2 of the 15 cases $(13 \%)$ of primary bladder adenocarcinoma, which is much lower than reported for urothelial carcinoma, ${ }^{34}$ and similar to the findings reported by Cowan et $a l^{7}$ however, unlike the latter study, tumor with both enteric as well as non-enteric (signet ring cell) morphology harbored TERT promoter mutation. This study clearly demonstrates the molecular heterogeneity in this rare and morphologically varied tumor. Therefore, the apparent variability of mutation frequency for TERT gene across prior studies and ours may not be surprising. Ours is the first study that 
demonstrates the relationship between TERT promoter mutation and genomic alterations in other important cancer genes. Both cases of primary bladder adenocarcinoma also harbored a PIK3CA mutation and one case (case 10) demonstrated TP53 and $A P C$ mutations. These combinatorial mutations may elicit the possibility of impact on biologic behavior or response to therapy; however, this small cohort and targeted sequencing data limits further insight.

KRAS mutations were identified in $20 \%(3 / 15)$ of primary bladder adenocarcinomas involving codons 12 and 13 (p.G12A, p.G12D and p.G13D). Alexander et $a l^{6}$ in their study also identified KRAS mutations, albeit at a lower frequency $(11.5 \%, 3 / 26$ primary bladder adenocarcinomas) that involved codons 12 (G12V) and 13 (G13D). In accordance to their study, KRAS mutations in our cohort were restricted to primary bladder adenocarcinomas with enteric-type morphology only. Alexander et $a l^{8}$ in a different study, also reported absence of EGFR alterations and $A L K$ rearrangement in primary bladder adenocarcinoma. Similarly, our study did not identify EGFR mutation or amplification in primary bladder adenocarcinoma.

The chief aim of prior studies in primary bladder adenocarcinoma has been an attempt to address the fundamental problem of distinguishing primary bladder adenocarcinoma from metastatic colorectal adenocarcinoma. $\beta$-catenin remains the only reliable discriminatory immunohistochemical marker in many but not all cases of primary bladder adenocarcinoma. This fundamental problem has not been approached from a molecular perspective. As a secondary aim of this study, we compared targeted genomic profile of primary bladder adenocarcinoma to stage appropriate colorectal adenocarcinoma and high-grade urothelial carcinoma without adenocarcinoma component. Many genomic alterations were overlapping between primary bladder adenocarcinoma and colorectal adenocarcinoma cohorts including major cancer genes such as $A P C, K R A S$, PIK3CA and TP53. In addition, genomic alterations in CTNNB1, FBXW7, and $I D H$ genes, identified in our primary bladder adenocarcinoma cohort, have been previously reported in colorectal cancers. ${ }^{38-40}$ When compared to high-grade urothelial carcinoma, the molecular alterations in primary bladder adenocarcinoma did demonstrate overlap for tumor suppressor genes TP53, RB1, and FBXW7 and oncogenes PIK3CA and KRAS. However, alterations in FGFR3 and HRAS genes, seen in our high-grade urothelial carcinoma cohort, were distinctly absent in primary bladder adenocarcinomas. Conversely, mutations in $A P C, C T N N B 1$, and $I D H 2$, seen in primary bladder adenocarcinoma, are uncommon to rare to highgrade urothelial carcinoma and were not identified in our high-grade urothelial carcinoma cohort. ${ }^{41,42}$

The small size of this cohort and the targeted genomic analysis precludes a definitive conclusion about the relationship of primary bladder adenocarcinoma with colonic adenocarcinoma or high-grade urothelial carcinoma. However, the findings raise an intriguing question about the origin of primary bladder adenocarcinoma. Prior studies, using immunohistochemistry, have suggested a close pathogenetic relationship between primary bladder adenocarcinoma (including precursor lesions) and colorectal adenocarcinoma. ${ }^{1,43}$ Broede et al investigated primary bladder adenocarcinoma and precursor lesions such as intestinal metaplasia and cystitis glandularis using a panel of immunohistochemical markers and suggested the possibility of divergent differentiation of primary bladder adenocarcinoma from multipotent urothelial cells given the common embryonic origin of the urogenital sinus and cloaca from the intermediate mesoderm. They demonstrated gradual change in immunohistochemical profile from cystitis glandularis through intestinal metaplasia to primary bladder adenocarcinoma. ${ }^{44}$ No molecular studies have been attempted to date to investigate this hypothesis. Alternatively, it can be hypothesized that primary bladder adenocarcinoma may arise from the embryonic rest of colorectal epithelium in the urinary bladder rather than urothelial cells. Conceptually, this will be similar to urachal tumors that are invariably adenocarcinomas arising from embryonic remnants of the allantoic duct and have been demonstrated to have a close embryonic, morphologic and immunohistochemical relationship with colorectal adenocarcinomas. ${ }^{45}$ The targeted approach of this current study is limited and comprehensive genomic profiling of this rare tumor is necessary to test these hypotheses.

In summary, using a targeted next-generation approach, we characterized primary bladder adenocarcinoma at a molecular level and identified several novel genomic alterations involving the MAP Kinase, mTOR, Wnt and tp53/rb1 pathways. While the genomic findings using this targeted approach are not diagnostically discriminatory between primary bladder adenocarcinoma and colorectal cancer, molecular testing of primary bladder adenocarcinoma is necessary since genomic alterations, such as the ones identified in our cohort, are potential therapeutic targets for personalized management of this aggressive tumor.

\section{Acknowledgments}

No external funding was used to support this research study.

\section{Disclosure/conflict of interest}

The authors declare no conflict of interest.

\section{References}

1 Rao Q, Williamson SR, Lopez-Beltran A, et al. Distinguishing primary adenocarcinoma of the urinary 
bladder from secondary involvement by colorectal adenocarcinoma: extended immunohistochemical profiles emphasizing novel markers. Mod Pathol 2013;26: 725-732.

2 Roy S, Smith MA, Cieply KM, et al. Primary bladder adenocarcinoma versus metastatic colorectal adenocarcinoma: a persisting diagnostic challenge. Diagn Pathol 2012;7:151.

3 el-Mekresh MM, el-Baz MA, Abol-Enein H, et al. Primary adenocarcinoma of the urinary bladder: a report of 185 cases. Br J Urol 1998;82:206-212.

4 Grignon DJ, Ro JY, Ayala AG, et al. Primary adenocarcinoma of the urinary bladder. A clinicopathologic analysis of 72 cases. Cancer 1991;67:2165-2172.

5 Wang HL, Lu DW, Yerian LM, et al. Immunohistochemical distinction between primary adenocarcinoma of the bladder and secondary colorectal adenocarcinoma. Am J Surg Pathol 2001;25:1380-1387.

6 Alexander RE, Lopez-Beltran A, Montironi R, et al. KRAS mutation is present in a small subset of primary urinary bladder adenocarcinomas. Histopathology 2012;61:1036-1042

7 Cowan ML, Springer S, Nguyen D, et al. Detection of TERT promoter mutations in primary adenocarcinoma of the urinary bladder. Hum Pathol 2016;53:8-13.

8 Alexander RE, Montironi R, Lopez-Beltran A, et al. EGFR alterations and EML4-ALK rearrangement in primary adenocarcinoma of the urinary bladder. Mod Pathol 2014;27:107-112.

9 Grasso C, Butler T, Rhodes K, et al. Assessing copy number alterations in targeted, amplicon-based nextgeneration sequencing data. J Mol Diagn 2015;17: 53-63.

10 Roy S, Durso MB, Wald A, et al. SeqReporter: automating next-generation sequencing result interpretation and reporting workflow in a clinical laboratory. J Mol Diagn 2014;16:11-22.

11 Wilson TG, Pritchett TR, Lieskovsky G, et al. Primary adenocarcinoma of bladder. Urology 1991;38:223-226.

12 Siefker-Radtke AO, Gee J, Shen Y, et al. Multimodality management of urachal carcinoma: the M. D. Anderson Cancer Center experience. J Urol 2003;169:1295-1298.

13 Galsky MD, Iasonos A, Mironov S, et al. Prospective trial of ifosfamide, paclitaxel, and cisplatin in patients with advanced non-transitional cell carcinoma of the urothelial tract. Urology 2007;69:255-259.

14 Hong JY, Choi MK, Uhm JE, et al. Palliative chemotherapy for non-transitional cell carcinomas of the urothelial tract. Med Oncol 2009;26:186-192.

15 Hatch TR, Fuchs EF. Intra-arterial infusion of 5-fluorouracil for recurrent adenocarcinoma of bladder. Urology 1989;33:311-312.

16 Logothetis CJ, Samuels ML, Ogden S. Chemotherapy for adenocarcinomas of bladder and urachal origin: 5fluorouracil, doxorubicin, and mitomycin-C. Urology 1985;26:252-255.

17 Kojima Y, Yamada Y, Kamisawa H, et al. Complete response of a recurrent advanced urachal carcinoma treated by S-1/cisplatin combination chemotherapy. Int J Urol 2006;13:1123-1125.

18 Teo M, Swan NC, McDermott RS. Sustained response of adenocarcinoma of the urinary bladder to FOLFOX plus bevacizumab. Nat Rev Urol 2011;8:282-285.

19 Vail E, Zheng X, Zhou M, et al. Telomerase reverse transcriptase promoter mutations in glandular lesions of the urinary bladder. Ann Diagn Pathol 2015;19: 301-305.
20 Dogruluk T, Tsang YH, Espitia M, et al. Identification of variant-specific functions of PIK3CA by rapid phenotyping of rare mutations. Cancer Res 2015;75: 5341-5354.

21 Gymnopoulos M, Elsliger MA, Vogt PK. Rare cancerspecific mutations in PIK3CA show gain of function. Proc Natl Acad Sci USA 2007;104:5569-5574.

22 Davies BR, Greenwood H, Dudley P, et al. Preclinical pharmacology of AZD5363, an inhibitor of AKT: pharmacodynamics, antitumor activity, and correlation of monotherapy activity with genetic background. Mol Cancer Ther 2012;11:873-887.

23 Paschka P, Schlenk RF, Gaidzik VI, et al. IDH1 and IDH2 mutations are frequent genetic alterations in acute myeloid leukemia and confer adverse prognosis in cytogenetically normal acute myeloid leukemia with NPM1 mutation without FLT3 internal tandem duplication. J Clin Oncol 2010;28:3636-3643.

24 Zhang C, Moore LM, Li X, et al. IDH1/2 mutations target a key hallmark of cancer by deregulating cellular metabolism in glioma. Neuro Oncol 2013;15:1114-1126.

25 Jardim DL, Wheler JJ, Hess K, et al. FBXW7 mutations in patients with advanced cancers: clinical and molecular characteristics and outcomes with mTOR inhibitors. PLoS One 2014;9:e89388.

26 Welcker M, Clurman BE. FBW7 ubiquitin ligase: a tumour suppressor at the crossroads of cell division, growth and differentiation. Nat Rev Cancer 2008;8:83-93.

27 Krieghoff E, Behrens J, Mayr B. Nucleo-cytoplasmic distribution of beta-catenin is regulated by retention. J Cell Sci 2006;119:1453-1463.

28 Miyoshi Y, Iwao K, Nagasawa Y, et al. Activation of the beta-catenin gene in primary hepatocellular carcinomas by somatic alterations involving exon 3. Cancer Res 1998;58:2524-2527.

29 Suriano G, Vrcelj N, Senz J, et al. beta-catenin (CTNNB1) gene amplification: a new mechanism of protein overexpression in cancer. Genes Chromosomes Cancer 2005;42:238-246.

30 Munemitsu S, Albert I, Souza B, et al. Regulation of intracellular beta-catenin levels by the adenomatous polyposis coli (APC) tumor-suppressor protein. Proc Natl Acad Sci USA 1995;92:3046-3050.

31 MacDonald BT, Tamai K, He X. Wnt/beta-catenin signaling: components, mechanisms, and diseases. Dev Cell 2009;17:9-26.

32 Henderson BR. Nuclear-cytoplasmic shuttling of APC regulates beta-catenin subcellular localization and turnover. Nat Cell Biol 2000;2:653-660.

33 Anastas JN, Moon RT. WNT signalling pathways as therapeutic targets in cancer. Nat Rev Cancer 2013;13: 11-26.

34 Kinde I, Munari E, Faraj SF, et al. TERT promoter mutations occur early in urothelial neoplasia and are biomarkers of early disease and disease recurrence in urine. Cancer Res 2013;73:7162-7167.

35 Nguyen D, Taheri D, Springer S, et al. High prevalence of TERT promoter mutations in micropapillary urothelial carcinoma. Virchows Arch 2016;469:427-434.

36 Zheng X, Zhuge J, Bezerra SM, et al. High frequency of TERT promoter mutation in small cell carcinoma of bladder, but not in small cell carcinoma of other origins. J Hematol Oncol 2014;7:47.

37 Zhong M, Tian W, Zhuge J, et al. Distinguishing nested variants of urothelial carcinoma from benign mimickers by TERT promoter mutation. Am J Surg Pathol 2015;39:127-131. 
38 Hartman DJ, Binion D, Regueiro M, et al. Isocitrate dehydrogenase-1 is mutated in inflammatory bowel disease-associated intestinal adenocarcinoma with low-grade tubuloglandular histology but not in sporadic intestinal adenocarcinoma. Am J Surg Pathol 2014;38:1147-1156.

39 Cancer Genome Atlas N. Comprehensive molecular characterization of human colon and rectal cancer. Nature 2012;487:330-337.

40 Muller MF, Ibrahim AE, Arends MJ. Molecular pathological classification of colorectal cancer. Virchows Arch 2016;469:125-134.

41 Millis SZ, Bryant D, Basu G, et al. Molecular profiling of infiltrating urothelial carcinoma of bladder and nonbladder origin. Clin Genitourin Cancer 2015;13: e37-e49.
42 Cancer Genome Atlas Research N. Comprehensive molecular characterization of urothelial bladder carcinoma. Nature 2014;507:315-322.

43 Sung MT, Lopez-Beltran A, Eble JN, et al. Divergent pathway of intestinal metaplasia and cystitis glandularis of the urinary bladder. Mod Pathol 2006;19: 1395-1401.

44 Broede A, Oll M, Maurer A, et al. Differential diagnosis of bladder versus colorectal adenocarcinoma: keratin 7 and GATA3 positivity in nuclear ss-catenin-negative glandular tumours defines adenocarcinoma of the bladder. J Clin Pathol 2016;69:307-312.

45 Gopalan A, Sharp DS, Fine SW, et al. Urachal carcinoma: a clinicopathologic analysis of 24 cases with outcome correlation. Am J Surg Pathol 2009;33: 659-668.

Supplementary Information accompanies the paper on Modern Pathology website (http://www.nature.com/ modpathol) 